\title{
Sobre el asco en la moralidad
}

\author{
ARLEEN L.F. SALLES \\ Centro de Investigaciones Filosóficas \\ Arleenl60@gmail.com
}

\begin{abstract}
Resumen: En este trabajo se aborda la cuestión del papel moral del asco. Se hace un bosquejo de esta emoción para luego concentrarse en la discusión sobre su estatus moral. Para ello, se identifican los tres argumentos principales contra su papel moral. El objetivo principal es mostrar que las objeciones más destacadas al asco moralizado no son lo suficientemente persuasivas, porque o bien tienden a basarse en casos en los que es evidente que el asco es indefendible, o bien parten de concepciones controvertidas sobre esa emoción y lo que representa. Para finalizar, se presentan algunas cuestiones planteadas por estudios recientes en psicología moral que tienden a vincular el asco con una cosmovisión conservadora de la moralidad.
\end{abstract}

Palabras clave: emociones, jerarquía, contaminantes, dignidad

\begin{abstract}
In this paper I focus on the moral role of disgust. After characterizing this emotion, I identify and critically examine three main objections recently presented to show that disgust is morally problematic. I argue that none warrant rejecting the moral appropriateness of disgust in all instances, for example, as a response to certain kinds of evil. I end by summarizing recent research in moral psychology that links the acceptance of disgust to a conservative view of morality.
\end{abstract}

Key words: emotions, hierarchy, contaminants, dignity

Los avances de la tecnología biomédica — desde la clonación de mamíferos y la investigación con células madre embrionarias hasta la creación de quimeras- han desatado una serie de controversias que giran en torno a la relevancia científica de estas prácticas y sus implicaciones morales, políticas y sociales. Estos avances también han revitalizado indirectamente una polémica dentro de la filosofía moral sobre el papel sociomoral que pueden desempeñar emociones negativas como el asco. En la discusión moral sobre la clonación, por ejemplo, o sobre la creación de quimeras, sus críticos frecuentemente invocan el carácter repulsivo de la práctica en cuestión (Cohen 2007, pp. 120-123). La clonación, nos dicen algunos, nos "enerva, nos da asco, nos horroriza, nos irrita" (Miller 1998, p. 81), y tal repulsión, según otros, constituye una especie de "alarma moral [...], expresión emocional de una sabiduría profunda, mas allá del poder de la razón de articularla" (Kass 1997, p. 20). Por ello consideran importante que los científicos continúen sintiendo repugnancia ante la idea de seguir adelante con sus in-

Diánoia, volumen LV, número 64 (mayo 2010): pp. 27-45. 
vestigaciones, "aun si no pueden articular sus razones" (Callahan 1997, p. 19).

¿Se puede asignar al asco algún tipo de papel moral?

En la primera parte de este trabajo haré un bosquejo de esta emoción; en la segunda identificaré y analizaré los tres argumentos principales presentados contra su papel moral. Mi objetivo principal es crítico, pues trato de mostrar que las objeciones más destacadas al asco moralizado no son lo suficientemente persuasivas porque o bien tienden a basarse en casos en los que es evidente que el asco es indefendible, o bien parten de concepciones controvertidas sobre esa emoción y lo que representa. Por último, me centraré en algunas cuestiones planteadas por estudios recientes en psicología moral que tienden a vincular una cosmovisión conservadora de la moralidad con el asco.

a) El asco. Ésta es una emoción compleja; presupone ideas sobre el carácter inferior, ofensivo y contaminante de un objeto o de una persona. Los psicólogos Paul Rozin y April Fallon definen lo que denominan "asco básico" [core disgust] como "repulsión ante la perspectiva de la incorporación oral de un objeto ofensivo" (Rozin y Fallon 1987, pp. 2341). Los autores toman como punto de partida el análisis evolucionista del asco que hizo Charles Darwin, quien afirmó que "el término asco, en su sentido más simple, significa algo ofensivo al gusto" (Darwin 1998, p. 257), y el análisis psicoanalítico de Andras Angyal que define el asco como "una reacción específica hacia desechos del cuerpo humano o animal" (Angyal 1941, p. 395).

Desde una perspectiva evolucionista, el asco se centra en el rechazo de alimentos y en el sentido del gusto. En cuanto omnívoros, los seres humanos tienen flexibilidad con respecto a qué consumir; pero por ello corren también el riesgo de consumir elementos contaminantes. Frente a esto, el asco se presenta como un mecanismo de supervivencia; es una respuesta primitiva fuerte y automática de rechazo hacia aquello que puede dañar o infectar. En su forma original está relacionado con alimentos, por lo cual se lo puede concebir como una suerte de guardián de la boca contra elementos animales que son potencialmente contaminantes (Rozin, Haidt y McCauley 1999).

Pese a que tiene precursores en animales no humanos que expresan disgusto frente a aquello que tiene mal sabor, el asco en los humanos va más allá del disgusto: posee un fuerte elemento cognoscitivo que no es provocado simplemente por ciertas características sensibles, sino que 
está relacionado de manera significativa con el "qué es" y "dónde ha estado", es decir, involucra creencias o evaluaciones en torno del objeto ofensivo (Rozin, Haidt y McCauley 1993). De acuerdo con Rozin y sus colegas, todas las instancias de asco señalan un significado común: la creencia en el carácter despreciable, contaminante y contagioso del objeto ofensivo. En ese sentido, es una emoción que involucra una especie de pensamiento jerarquizante.

Según Rozin y sus colegas, el "asco elaborado" es una reacción de rechazo a eventos que "nos hacen recordar nuestra naturaleza animal" (Rozin 1997; Haidt, Rozin, McCauley e Imada 1997). Estos autores afirman que los seres humanos necesitan esconder su naturaleza animal bajo un velo de prácticas y rituales que los humaniza, lo cual explicaría por qué ciertos actos sexuales, la falta de higiene, la muerte y la mutilación corporal generalmente provocan asco (Rozin, Haidt y McCauley 1993, p. 584). En estos casos, el asco estaría cumpliendo la función de humanizarnos, asumiendo el papel de guardián del yo corporal, rechazando elementos que violan "el templo del cuerpo" y trazando una distinción entre lo animal y lo humano. ${ }^{1}$

El llamado "asco moralizado" es el provocado por ofensas y transgresiones sociomorales. Existen dos teorías respecto de la naturaleza de este tipo de reacción. Para Rozin, Haidt y sus colegas, pese a que el asco comenzó como un mecanismo de defensa del cuerpo y originariamente lo provocaban contaminantes animales específicos, se ha transformado y puede ser ocasionado por elementos desvinculados de lo orgánico y animal; por ejemplo, por nociones abstractas como la violación de reglas morales. Mucha gente se reconoce asqueada por el racismo, por la tortura o por actos de violencia injustificada. Según estos autores, en tales casos el asco estaría asumiendo el papel de protector de la dignidad humana en el orden social; es un modo de rechazo de marcos valorativos considerados degradantes y diferentes del propio.

El psicólogo Paul Bloom, en cambio, considera que el asco siempre es una respuesta instintiva a desencadenantes específicos. Para Bloom, el verdadero asco tiene que ver con lo sensual; es provocado por lo carnal, por actos corporales y por los seres que los ejecutan. Por ello - afirma-, cuando se utiliza el discurso del asco para discutir conductas humanas inmorales, se lo hace de manera puramente metafórica; en verdad estas conductas no son asquerosas en sí, porque carecen de las características físicas y carnales esenciales para serlo (Bloom 2004).

${ }^{1}$ Para una perspectiva diferente, menos conceptual, véase Bloom 2004. 
b) El asco y la moralidad. Sea provocado por nociones abstractas acerca de la violación de derechos, sea producto de desencadenantes carnales específicos, el asco parece funcionar como un medio importante para internalizar prohibiciones morales. La mayoría de las sociedades humanas repudian algunos tipos de comportamiento que consideran potencialmente contaminantes, y el esquema del asco ha sido adaptado o aplicado metafóricamente a estas formas de rechazo. Como afirma William Ian Miller en su análisis: "seamos puritanos o no, expresamos muchos de nuestros juicios morales básicos utilizando el lenguaje del asco" (Miller 1997).

Respecto de la justificabilidad del asco moralizado, se pueden distinguir tres líneas argumentales. La primera línea parte de la base de que el único fundamento apropiado para el juicio moral es el intelecto y que lo emocional no puede ni debe desempeñar papel alguno en el razonamiento moral. Según este planteamiento, por ser el asco una emoción, no es moralmente justificable.

Esta perspectiva adquiere matices diferentes según la concepción aceptada sobre la naturaleza de las emociones en general. De acuerdo con la teoría fisiologista de fines del siglo XIX, las emociones son meras sensaciones, experiencias de cambios fisiológicos sin complejidad o contenido conceptual (James 1884). Si esto fuera cierto, las emociones no serían susceptibles de la influencia de la razón, lo cual respaldaría la idea de que no pueden cumplir papel alguno en la moralidad.

Sin embargo, esta versión de la postura fisiologista sobre la naturaleza de las emociones enfrenta problemas; entre ellos, cómo explicar la aparente inteligibilidad de lo emocional y cómo entender el papel que las emociones cumplen en la justificación de las acciones. En las últimas décadas se han propuesto análisis diferentes de la naturaleza de las emociones que intentan dar respuesta a algunas de estas cuestiones. Desde el cognoscitivismo, por ejemplo, se argumenta que lejos de ser meros sentimientos asociados a cambios fisiológicos, las emociones necesariamente involucran un juicio determinado (Solomon 1976, 1993; Nussbaum 2001). Perspectivas híbridas tienden a sostener que las emociones son sentimientos compuestos por una dimensión evaluativa (no necesariamente reducible a un juicio) y una dimensión afectiva (Greenspan 1988). ${ }^{2}$ Aun quienes aceptan el fisiologismo reconocen que éste debe ser revisado, puesto que "las emociones no son simplemente percepciones del cuerpo sino también percepciones de nuestras relaciones con el mundo" (Prinz 2004, p. 20). En suma, aunque se trate de

${ }^{2}$ Olbeth Hansberg (1998) pasa revista a alguna de estas críticas.

Diánoia, vol. LV, no. 64 (mayo 2010). 
diversas posturas cognoscitivistas, de posturas híbridas o de posturas neofisiologistas, existe acuerdo en que las emociones desempeñan un papel mucho más importante en la moralidad que el que típicamente se les atribuye. ${ }^{3}$

La segunda estrategia respecto de la justificabilidad moral del asco no basa su rechazo de esta emoción en un repudio general de las emociones, sino que se concentra en ciertas características específicas que harían del asco una emoción moralmente cuestionable. Este enfoque es el sugerido por Martha Nussbaum en su valiosa discusión sobre el papel del asco en el discurso público (Nussbaum 1999, 2001, 2004). ${ }^{4}$ Pese a que la preocupación fundamental de Nussbaum es evaluar el papel del asco en la ley, su discusión necesariamente toca el papel que cumple el asco moralizado.

Nussbaum defiende una postura cognoscitivista de las emociones. Argumenta que no se deben rechazar las emociones como clase, puesto que constituyen modos de reflexión importantes; pero debemos evaluar las cogniciones que cada una involucra para analizar su confiabilidad, su formación y sus manifestaciones, y sobre la base de estas consideraciones, determinar qué papel deben desempeñar en el discurso público y, presumiblemente, qué estatus moral se les puede asignar. Para Nussbaum el asco moralizado pertenece al grupo de emociones que son “altamente problemáticas" per se (Nussbaum 2004, p. 105).

Finalmente, la tercera de las líneas argumentales considera que el asco está moralmente justificado, por lo menos en ciertas circunstan-

${ }^{3}$ Por otro lado, no todo rechazo de lo emocional en la moralidad debe ser vinculado con un fisiologismo simplista respecto de la naturaleza de las emociones. Los estoicos argumentaban que debemos desembarazarnos de las emociones porque están basadas en juicios falsos acerca de lo que es bueno y malo: nos llevan a sobrevalorar cosas externas, promoviendo vulnerabilidades que son incompatibles con la virtud. Concebidas así, las emociones no pueden ser racionalmente controladas, puesto que son de por sí una forma de racionalidad corrupta, por lo cual se las debe extirpar de la vida moral. Este tipo de postura plantea cuestiones significativas sobre qué es una vida valiosa y qué tipo de vulnerabilidades son compatibles con la estabilidad que, al parecer, es un requisito de la moral, pero está fundada en afirmaciones descriptivas sobre el razonamiento moral y en proposiciones normativas sobre el valor de la autosuficiencia y de la indiferencia afectiva que han sido blanco de agudas críticas, especialmente en los últimos años. Sobre la base de estudios empíricos en torno al juicio moral y sobre su riqueza y plasticidad, un número creciente de filósofos morales propugna formas de reflexión moral más ricas. Estos pensadores critican las perspectivas puramente racionalistas y cuestionan el ideal de apatía que esta postura estoica recomienda.

${ }^{4}$ Ésta es también la postura sugerida por Bloom (2004). 
cias. A continuación me centraré en los puntos más importantes planteados por la segunda y la tercera posturas.

Nussbaum presenta tres consideraciones fundamentales para mostrar que el asco es moralmente problemático.

a) El asco no es una emoción confiable. Respecto de cualquier actitud emocional, puede comenzarse haciendo la siguiente pregunta: ¿está justificada por la evidencia que la provoca? Si la respuesta es afirmativa podemos decir, junto con Patricia Greenspan, que la emoción es representacionalmente racional (Greenspan 1988). Una emoción es representacionalmente racional cuando refleja una percepción significativa; es decir, en la medida en que se corresponde con algún tipo de evidencia que la causa. Esto significa que una emoción puede ser apta o apropiada en lo que podríamos llamar un sentido meramente cognoscitivo.

Ahora bien, comencemos con el asco no moral. En numerosos casos constituye una reacción a la presencia de verdaderos contaminantes en el objeto que la provoca; sin embargo, existen instancias en las que es representacionalmente irracional. Tal sería el caso cuando está basada en "pensamiento mágico" y no en un peligro real. Rozin utiliza el término "pensamiento mágico" para referirse al salto injustificado que se da de contaminantes reales (que justificarían la presencia de la emoción) a cosas que, si bien son similares al objeto asqueroso, en verdad no poseen en sí mismas atributos asquerosos. El asco es un tipo de emoción que puede transmitirse de un objeto a otro por medio del mecanismo de contaminación psicológica, mediado por la "ley de contagio" y la "ley de la similitud" (Rozin, Haidt y McCauley 1999).

De acuerdo con la ley de contagio, cosas que han estado en contacto continúan afectándose (Rozin, Haidt y McCauley 1999). La idea operativa es que los objetos poseen una "esencia" o cualidad inmaterial que se transfiere por medio del contacto. Por ello, lo que importa no es verdaderamente la presencia de elementos contaminantes en el objeto que provoca asco, sino su historia de contacto con lo contaminado (Rozin, Haidt y McCauley 1993, p. 583). ${ }^{5}$

${ }^{5}$ Esto explicaría por qué, mas allá de las características sensibles, un número considerable de personas responde con asco frente a un vaso de agua totalmente esterilizada y pura, pero que previamente contuvo vómito de perro.

Diánoia, vol. LV, no. 64 (mayo 2010). 
De acuerdo con la ley de la similitud, "si dos cosas son superficialmente semejantes, se asemejan también en un sentido más profundo" (Rozin, Haidt y McCauley 1993, p. 583). Este mecanismo psicológico explicaría por qué se consideran repugnantes objetos que pueden tener una apariencia repulsiva pero que en verdad no lo son. Lo crucial es la asociación que se hace entre el objeto y la sustancia ofensiva aun cuando el objeto en sí no sea repugnante. ${ }^{6}$

Ahora bien, si el asco básico puede tener un origen tan idiosincrásico, es de esperarse que pase algo semejante con el asco moralizado. Y esto es lo que Nussbaum afirma. Primero observa que se puede sentir asco en casos en los que no existe peligro o transgresión moral alguna; para ilustrar esto señala que "la mera presencia de personas mental o físicamente discapacitadas [...] frecuentemente ha provocado asco; y sin embargo es muy difícil sostener que constituyan un peligro para la comunidad" (Nussbaum 2004, p. 79). De manera inversa, pueden existir trangresiones morales que no provoquen sentimientos de asco (Nussbaum 2004, p. 84). De esto se podría concluir que el hecho de que una persona sienta un "malestar visceral" frente a determinados actos, prácticas o incluso frente a otros seres humanos no es indicativo de que tal malestar esté justificado. Y si esto es cierto, el asco resultaría ser una emoción muy poco confiable desde el punto de vista moral.

Pero, además, para Nussbaum no se trata tan sólo de la escasa confiabilidad de la emoción: la autora señala que la experiencia muestra que el asco puede ser y ha sido proyectado a ciertos grupos y personas con consecuencias escalofriantes. Todo lo que se necesita para sentir asco por otros es reforzar la asociación entre ellos y cualidades repugnantes; una vez que el asco está presente, no es difícil tratar a otros de manera inhumana y degradante (Nussbaum 2004, Bloom 2004). El papel que históricamente ha desempeñado el asco, propiciando inequidades y prejuicios moralmente injustificados, haría de ésta una emoción potencialmente siniestra.

Aunque las consideraciones planteadas por Nussbaum son valiosas, no muestran de manera concluyente que el asco no debe cumplir papel moral alguno. En primer lugar, el problema de la "poca confiabilidad" no es exclusivo de emociones como el asco: es evidente en numerosas emociones, incluida la ira que Nussbaum acepta como moralmente apropiada. Si el problema del asco radica en que en ciertas ocasiones no está justificado por la evidencia y en que puede ser generado ar-

${ }^{6}$ Rozin y sus colegas citan como ejemplo el hecho de que la mayoría de las personas se rehúsan a ingerir chocolate que luce como material fecal. 
bitrariamente, habría que ser igualmente escéptico respecto del papel que las emociones en general, y ciertas emociones en particular, tienen en la moralidad, dado que existen instancias es las que éstas tampoco están justificadas por la evidencia. Es fácil imaginar casos en los que la ira es representacionalmente irracional, o en las que es transmitida por contagio (piénsese en el mecanismo que opera en casos de violencia callejera) o en los que causa daños serios a numerosas personas. Dada la aceptabilidad moral, según Nussbaum, de emociones que también pueden operar por medio de estos mecanismos, que el asco opere así no parece ser motivo suficiente para sugerir que, por ende, es una emoción más problemática que otras desde el punto de vista moral. ${ }^{7}$

No se trata de que el asco sea una emoción fácilmente justificable; de hecho, quienes desean otorgarle algún tipo de papel moral conceden que el asco puede ser provocado por "asociaciones arbitrarias, o por falta de familiaridad, o por mera repulsión física” (Midgley 2000, p. 9). Lo que plantean, sin embargo, es si debemos concluir por ello que el asco es siempre poco confiable y siempre moralmente problemático. $\mathrm{Y}$ frente a esto responden de manera negativa, proponiendo en cambio una actitud no de rechazo de la emoción, sino de crítica y análisis. Mary Midgley afirma que, respecto del asco, "siempre debemos mirar bajo la superficie. Debemos explicitar el mensaje de nuestras emociones y ver qué nos tratan de decir" (Midgley 2000, p. 9); pero, al mismo tiempo, considera que existen motivos para cuestionar el carácter moral de quien no siente indignación, asco o ira acerca de prácticas como la esclavitud y la tortura.

Ahora bien, es evidente que el asco ha sido mal utilizado en numerosas ocasiones. Un repaso de la historia mundial hace evidente la estigmatización y el maltrato padecido por numerosas personas pertenecientes a distintos grupos étnicos o sociales a las cuales en algún momento se asoció sistemáticamente con características asquerosas (Nussbaum 2004, pp. 107-115). No hay duda de que toda acción basada en estas instancias injustificadas de asco es claramente inmoral... Pero la pregunta es: ¿debemos concluir, por lo tanto, que no hay transgresión moral alguna frente a la cual el asco sea la reacción apropiada?

Creo que la respuesta es negativa. De hecho, si se quiere argumentar que el asco es moralmente inapropiado, se lo debe hacer tomando

${ }^{7}$ Es decir, si la ocasional arbitrariedad de su generación es motivo para descalificarlo moralmente, también lo es para descalificar moralmente otras emociones que, no obstante, filósofos como Nussbaum aceptan como potencialmente significativas desde el punto de vista moral.

Diánoia, vol. LV, no. 64 (mayo 2010). 
como punto de partida casos en los cuales parece prima facie justificado. Puede que estos casos no sean demasiado frecuentes, pero sin duda existen acciones que son moralmente aberrantes, actos de injusticia extrema y de crueldad intencional (Glover 2002). En estos casos, el asco no parece una emoción inadecuada en el sentido de que "se ajusta a la evidencia". ¿Por qué no decir, entonces, que en esos casos la reacción es moralmente apropiada? Si el asco puede ocasionalmente ser representacionalmente racional, la negación de su valor moral debe responder a otros motivos. A ellos me dedicaré a continuación.

b) El asco no tiene valor instrumental. Según este tipo de objeción, aun cuando el asco moralizado a veces estuviera justificado por la evidencia, es una emoción a la cual la gente puede renunciar, y probablemente debe hacerlo, porque no es productiva ni moralmente adaptativa.

Una emoción puede ser moralmente productiva o instrumentalmente valiosa en dos sentidos. En primer lugar, en cuanto que otorga información moralmente significativa; en segundo lugar, en cuanto que fomenta un tipo determinado de comportamiento moral. La ira, por ejemplo, es una emoción que ha sido moralmente justificada sobre la base de estas consideraciones: potencialmente, puede hacer que el sujeto reconozca que se encuentra en una situación injusta (por lo cual se considera que la emoción en sí provee información moralmente relevante), y puede llevar a comportamientos productivos, motivando a actuar de manera tal que se corrijan injusticias. El mismo tipo de estrategia se utiliza para tratar de justificar moralmente otras emociones negativas como la amargura.

Respecto del asco, es relativamente fácil concordar en que brinda algún tipo de información. El tema que se plantea, sin embargo, es el de si esta información es moralmente relevante, es decir, si es una manifestación de sabiduría o de ignorancia moral y, por ende, si debe ser tomada en cuenta (Nelson 2003, p. 134). Algunos pensadores consideran que la respuesta es afirmativa. El filósofo John Kekes, por ejemplo, considera que el asco implica el reconocimiento de que ciertos tabúes morales han sido violados y de que se ha llegado al límite de la tolerancia (Kekes 1992). Leon Kass sostiene que "la repugnancia [...] se revela contra los excesos de la voluntad humana, advirtiéndonos que no debemos transgredir lo que es terriblemente profundo" (Kass 1997, p. 20). Miller considera que el asco señala aquellas cuestiones morales sobre las cuales no debemos aceptar compromiso. Para Miller (1997), la presencia de la emoción nos muestra que estamos verdadera y profundamente comprometidos con una norma que está siendo violada. Dan 
Kahan retoma el razonamiento de Miller y afirma que el asco es necesario para percibir el carácter excepcionalmente abominable de ciertos comportamientos (Kahan 1999).

Nussbaum, en cambio, argumenta que la información que el asco brinda es o bien innecesaria, o bien moralmente inapropiada. Es innecesaria en tanto "no necesitamos recurrir al asco para que nos informe que el asesinato y la crueldad son incorrectos" (Nussbaum 2004, p. 84); en ese sentido, el asco podría ser eliminado del repertorio de reacciones moralmente relevantes. Pero Nussbaum apunta más lejos. No se trata sólo de que el asco no aporte información moralmente valiosa: Nussbaum considera que el asco aporta información que es moralmente problemática en sí.

Nussbaum parte de la idea de Rozin de que el asco expresa rechazo de la propia animalidad, pero agrega algo más. Para Nussbaum, el asco no rechaza simplemente lo animal, dado que existen características animales - como la fuerza y la rapidez- que los seres humanos admiran. Lo que el asco rechaza es "el tipo de vulnerabilidad que compartimos con otros animales, la propensión a descomponernos y convertirnos en residuos" (Nussbaum 2004, p. 92). Nussbaum está convencida de que los seres humanos tratamos de escondernos de nuestras vulnerabilidades y que "en el proceso desarrollamos y enseñamos a sentir vergüenza por la fragilidad humana y asco frente a signos de animalidad y mortalidad" (Nussbaum 2004, p. 336). En ese sentido, el asco "gira alrededor del deseo de ser el tipo de persona que uno no es, a saber, no animal e inmortal" (Nussbaum 2004, p. 102). En la medida en que intenta ocultar la propia vulnerabilidad humana, marcando límites y señalando contaminación, distrae de las cuestiones que son genuinamente importantes en la moralidad: el daño, la injusticia, la subordinación y la opresión.

Por otro lado, la concepción del asco de Nussbaum pone en evidencia por qué considera que esta emoción tampoco es prácticamente adaptativa. Quienes argumentan a favor del papel moral del asco consideran que aun si esta emoción no desempeñara un papel activo en la promoción de la acción moral, por lo menos cumple uno vital en ayudar a evitar el comportamiento inmoral. Sostienen que una vida moralmente satisfactoria requiere no sólo sentimientos de empatía, benevolencia y compasión que incentiven el comportamiento moral, sino también el deseo de evitar la crueldad. Y el asco, precisamente por su carácter jerarquizante, podría ser particularmente apropiado para esto funcionando como una especie de mecanismo de autorreconocimiento y autopro- 
tección (Kekes 1992). ${ }^{8}$ En cambio, para Nussbaum la propia naturaleza del asco lo hace una emoción moralmente paralizante; en cuanto que nos confronta con la vulnerabilidad humana, no lleva a la acción sino al escape. Por ello sostiene que debe ser superado si se quiere lograr un sentimiento de empatía genuino y moralmente constructivo (Nussbaum 2004, p. 106).

La plausibilidad de esta objeción depende en gran medida de que se acepte su convicción de que el asco es efectivamente una emoción de rechazo de la propia animalidad y que abarca "deseos imposibles de pureza, inmortalidad y no animalidad" (Nussbaum 2004, p. 14). Y el problema es que la postura de Nussbaum no está lo suficientemente fundamentada en esto. El tema que pide un análisis más cuidadoso es, entonces, ¿en verdad esconde el asco nuestra humanidad, como Nussbaum afirma? ¿Expresa siempre rechazo a lo que somos? ¿No podría argumentarse que en algunos casos expresa lo contrario, que justamente la reacción de asco es una manera de expresar hasta qué punto nos sentimos humanos y vulnerables? En verdad, Nussbaum misma parece ambivalente al respecto. Mientras que, por un lado, considera lesivo el asco por esconder nuestra humanidad, por el otro sugiere que la existencia de esta reacción emocional es una muestra esencial de humanidad, dado que "pedir que los seres humanos no eviten la descomposición o su temor a la muerte es posiblemente pedirles que no sean humanos" (Nussbaum 2004, p. 121).

Sin duda, el asco implica rechazo hacia algo, pero no está claro que en todos los casos implique rechazo de la propia corporalidad y de su vulnerabilidad, y por ello no resulta evidente que el asco no pueda ser una emoción "constructiva" en algún sentido. El asco como reacción de rechazo de comportamientos moralmente atroces puede ser vinculado con la propia identidad moral de la persona, y podría ser reivindicado como impulso importante para la reflexión y el desarrollo moral. Es decir, en un mundo en el que las personas son moralmente iguales y vulnerables unas con respecto a otras, y donde la crueldad intencional es una realidad, la presencia del asco puede reforzar el sentido de identidad y compromiso moral propio. La reacción de asco provocada por ciertos comportamientos, o incluso por el carácter de ciertas personas, puede motivar al agente a examinar con más detenimiento sus

${ }^{8}$ Haidt (2003) nota que el asco es una emoción pro-social; en cuanto que conduce a apartarse de aquellos cuyos comportamientos generan asco, intenta funcionar como fuerza disuasoria respecto de comportamientos que son moralmente inapropiados.

Diánoia, vol. LV, no. 64 (mayo 2010). 
compromisos morales y su identidad moral, otorgando una perspectiva más completa del tipo de valores morales que avala y que lo definen. ${ }^{9}$

c) La objeción liberal. La objeción más seria y filosóficamente interesante puede ser llamada la "objeción liberal". Como hemos visto, el asco está vinculado con la creencia o evaluación de que algo o alguien es inferior o contaminante. Ahora bien, a criterio de Nussbaum, este tipo de evaluación negativa es inapropiada en general y moralmente peligrosa en particular. Su razonamiento es el siguiente: además de ser una emoción que nos lleva al autoengaño, el asco no hace una distinción clara entre el carácter repulsivo de un acto determinado y la persona que lo comete. En ese sentido, la presencia de esta emoción tiene un impacto directo sobre el valor moral que asignamos a las personas (Nussbaum 2004, p. 166). Observa que "la ira ante un acto malo es compatible con el deseo de rehabilitar a quien lo perpetró y con el respeto a la dignidad humana de quien lo perpetró. El asco básicamente quiere que la persona desaparezca" (Nussbaum 2004, p. 106). Por ello, el tipo de jerarquización inherente en el asco "representa una amenaza a la idea de igual valor y dignidad de las personas, elemento importante del tipo de moralidad que la mayoría de las personas defendería" (Nussbaum 2001, p. 221). Para Nussbaum, al demarcar y hacer distinciones, el asco nos llevaría a ver a quien comete actos moralmente aberrantes como un monstruo fuera de los límites de nuestro propio universo moral. Generaría, por ello, dos actitudes cuestionables: la autoindulgencia, en cuanto que dificultaría el tipo de autoexamen necesario para reconocer "la presencia del mal en nosotros mismos" (Nussbaum 2001, pp. 451452), y, por otro lado, la de autocomplacencia, que nos hace creer que nosotros mismos somos incapaces de cometer actos de crueldad extrema. Como consecuencia, en el planteamiento de Nussbaum el asco moralizado se constituiría en un impedimento para el desarrollo de una conciencia moral individual sana y de una sociedad liberal donde se pudiera erradicar la maldad, puesto que impide reconocer que quien comete actos bárbaros es un ser humano como cualquier otro y que todos los seres humanos son capaces de asumir un comportamiento cruel.

${ }^{9}$ Glover sugiere que la identidad moral de las personas puede ser sistemáticamente erosionada de manera tal que lleguen a cometer actos de los que nunca se habrían creído capaces de cometer. Y, sin embargo, en su análisis de cómo los nazis ejecutaron sus acciones sugiere que si una persona considera que un comportamiento particular es moralmente repugnante, no es tan fácil entrenarla para que lo ejecute. Esto sugiere que es improbable que sentir sentimientos de asco facilite comportamientos repugnantes en uno mismo.

Diánoia, vol. LV, no. 64 (mayo 2010). 
Ahora bien, para empezar, este argumento toma como punto de partida la concepción (comentada en el apartado anterior) de que el asco es una emoción que involucra el "deseo vano" de inmortalidad y no animalidad, que implica no reconocer las limitaciones inherentes a la corporalidad y los diferentes sentidos en los cuales somos vulnerables. Ya hice notar que Nussbaum no provee argumentos demasiado persuasivos en defensa de esta idea; pero habiendo hecho esa salvedad, se puede decir que la objeción de Nussbaum genera otros problemas. El primero es que, aunque el asco no siempre implique una visión distorsionada de la propia humanidad, como Nussbaum cree, es claramente una emoción de rechazo. ¿Se sigue, entonces, que es necesariamente incompatible con la actitud moral? ¿Implica su presencia que uno se considera moralmente perfecto?

Comencemos por la supuesta injusticia que supondría el considerar que quienes ejecutan acciones moralmente aberrantes son monstruos morales. De acuerdo con la autora, el asco es moralmente inapropiado precisamente porque es un tipo de emoción jerarquizante, que no puede sino violar la regla moral de que las personas merecen igual respeto y dignidad. Pero ¿qué significa esto? Nussbaum en realidad parece sugerir que del mero hecho de que las personas poseen dignidad se sigue que no se las puede juzgar como moralmente inferiores, aun cuando tal juicio tome como punto de partida sus propias acciones. Para ella, argumentar lo contrario manifiesta un compromiso moral con una postura no liberal. Pero esto es controvertido. Como nota Kahan, toda moralidad, incluida una de corte liberal, hace y necesita un ordenamiento moral (Kahan 1999). Decir que las acciones de $X$ son moralmente mejores que las de $Y$ supone dicho ordenamiento moral, un posicionamiento dentro de una escala de valores, así como lo supone decir que $X$ es mejor persona que $Y$; de hecho, se podría argumentar que algún tipo de jerarquización moral puede ser no sólo moralmente permisible, sino hasta necesaria para mostrar respeto genuino por la agencia moral de las personas, por sus decisiones autónomas y sus acciones. En suma, no está claro que una actitud jerarquizante sea siempre intrínsecamente incorrecta. ${ }^{10}$ Por ello, la creencia de que el juicio moral y emocional de que ciertos comportamientos o personas son moralmente rechazables y

${ }^{10}$ Existen instancias claras de jerarquización moral injustificada; por ejemplo, cuando está basada en características moralmente irrelevantes de las personas en cuestión. Aquí, sin embargo, estoy interesada en la cuestión de la jerarquización moral cuando se toman en serio las acciones de personas a las cuales se les reconoce la capacidad de elegir y actuar sobre la base de sus elecciones. 
contaminantes es siempre problemática es por lo menos controvertida $\mathrm{y}$, por ende, requiere una mejor justificación. ${ }^{11}$

Por otro lado, no es autoevidente que la jerarquización presente en el asco implique que uno se considera moralmente invulnerable o perfecto. El rechazo del comportamiento o de la personalidad moral de otros puede interpretarse de manera diferente, como un reconocimiento de dos cosas: la propia vulnerabilidad y que la inmoralidad admite grados. Respecto de lo primero, hemos visto que es verosímil pensar que el asco moralizado intenta proteger al ser humano de cosas frente a las cuales éste es particularmente vulnerable. Respecto de lo segundo, es importante notar que reconocer la presencia de inmoralidad en otros no implica que uno se conciba sin deficiencias morales. Para ilustrar esto: que $X$ tenga una tendencia a mentir la hace en parte moralmente deficiente; sin embargo, este hecho no invalida moralmente el asco que puede sentir frente al violador sádico o al nazi que habla con indiferencia del exterminio de millones de personas. De nuevo, no se trata de afirmar que $X$ no posee falla moral, sino de que $X$ puede autorreconocerse como persona moralmente imperfecta $\mathrm{y}$, al mismo tiempo, tener una fuerte reacción emocional de rechazo a la crueldad intencional o el sadismo.

Por último, es problemático que Nussbaum invoque la noción misma de "dignidad humana" para argumentar contra el asco. Ésta es una noción poco clara; ${ }^{12}$ se utiliza comúnmente como fundamentación moral de posturas diversas: de hecho, tanto Kass como Nussbaum la mencionan en sus respectivos análisis. Está claro, sin embargo, que no existe acuerdo sobre qué significa y qué implica respetarla. La noción de dignidad humana en Kass parece estar más fuertemente influida por su compromiso con una cosmovisión según la cual lo "natural" merece un respeto especial. Por otro lado, aunque la noción de dignidad operativa no es explícita en el análisis de Nussbaum, se insinúa como algo diferente: la autora parece inclinarse por la idea de que la dignidad humana tiene que ver con el ejercicio de las capacidades humanas, pero al mismo tiempo sugiere que respetar la dignidad implica tanto el reconocimiento de tales capacidades como de sus fragilidades y vulnerabilidades qua humanos. Dada la indeterminación de la noción, es dudoso que pueda cumplir el papel moral que Nussbaum pretende en este contexto. La noción de dignidad debe ser cuidadosamente deba-

${ }^{11}$ Para un análisis más profundo de esta cuestión, véase "On Disgust and Human Dignity" de Inmaculada De Melo Martin y Arleen Salles (inédito).

${ }^{12}$ Para un análisis de este tema véase Hayry 2004. 
tida, y no utilizada como eslogan moral para poner punto final a la discusión.

a) El asco: ¿la emoción conservadora? En la antropología se ha argumentado que existen por lo menos tres códigos éticos que distintas sociedades, con organizaciones sociopolíticas y estructuras productivas diversas, exhiben (Shweder et al. 1997). El código ético de la autonomía (que se correspondería con una moralidad de corte liberal) subraya fundamentalmente conceptos regulativos como la prevención de daños, el respeto a los derechos y el tratamiento justo. Dentro de esta concepción, el objetivo básico de la moralidad es proteger el espacio de la elección personal, la voluntad, la libertad y la responsabilidad de la persona, y promover el ejercicio de la voluntad individual. El código moral de la comunidad (generalmente conocido como "moralidad comunitarista") requiere obediencia a las autoridades, respeto a los valores asumidos colectivamente, y acciones consistentes con el papel social que cada uno cumple. El objetivo básico de este discurso es proteger la integridad moral de los roles que constituyen una sociedad o una comunidad. Por último, el código de la divinidad (que se correspondería con una moralidad conservadora) enfatiza los conceptos de orden sagrado, tradición, santidad, pecado y polución. El ser es concebido de manera espiritual, conectado con el orden natural de las cosas. Los actos que pueden degradar la propia naturaleza espiritual son condenados, independientemente de si producen daño a otros. El objetivo de este código es proteger el alma y el espíritu de la polución moral.

Lo anterior no implica que los rasgos de cada código siempre se manifiesten con la misma intensidad en todas las sociedades, ni que los códigos no puedan coexistir en distintas sociedades (Shweder et al. 1997). Pero por lo general uno de los códigos tiende a prevalecer sobre los otros en distintas sociedades (por ejemplo, en el caso de las sociedades occidentales, el de la autonomía tiene un papel más evidente que el de la divinidad o el de la comunidad).

Rozin y sus colegas han tratado de mostrar que existe una alianza entre el enojo y la violación del código de la autonomía, el desprecio y la violación del código de la comunidad, y el asco y la violación del código de la divinidad (Rozin, Lowery, Imada, Haidt 1999). Reconocen que la correspondencia entre estas emociones y la violación de los códigos respectivos no es perfecta; sin embargo, consideran que, en general, en cualquier cultura las acciones que violan la ética de la 
autonomía muy probablemente produzcan enojo (emoción a la cual se vincula con el reclamo de derechos), las violaciones de la ética de la comunidad muy probablemente produzcan desprecio (vinculado con el respeto por los otros), y las violaciones de la ética de la divinidad muy probablemente produzcan asco (Rozin, Lowery, Imada, Haidt 1999). La posible vinculación entre el asco y perspectivas morales que tienden a centrarse en nociones asociadas con el código de la divinidad continúa siendo uno de los temas de investigación del psicólogo moral Jonathan Haidt. En artículos recientes, Haidt y sus colegas notan que quienes se reconocen como moralmente conservadores viven una cultura moral particular, con un esquema conceptual que los lleva a aceptar la legitimidad de ciertas reacciones emocionales que los liberales tratan de no tomar en cuenta (Haidt y Hersch 2001; Haidt y Graham 2007, y Graham, Haidt y Nosek 2009).

Si se tienen en cuenta estos estudios, la discusión sobre el papel moral del asco resulta más fascinante. En verdad, un pensador como Kass, que invoca el asco para evaluar algunas prácticas morales, en general tiene una tendencia a utilizar un vocabulario que se puede asociar con una perspectiva conservadora. Sus palabras se encuadran muy claramente dentro de una determinada cosmovisión en la que consideraciones sobre el daño y el sufrimiento de otros se distinguen de otras que tienen que ver con "el orden natural de las cosas" y el respeto a tradiciones morales, y en la que es precisamente la violación de tal orden natural y de tales tradiciones lo que produce repulsión, no las consideraciones sobre daños.

Nussbaum misma considera que el asco es incompatible con una moralidad de corte liberal (Nussbaum 2004). Ahora bien, no se puede decir que Nussbaum posea una concepción moral anémica o minimalista. Su visión de la moralidad incluye respeto mutuo y reciprocidad, la idea de que los seres humanos son fines y no medios, que se debe promover su agencia moral, se deben valorar las relaciones personales y facilitar que ejerciten sus capacidades y que vivan una vida plena (Nussbaum 2001). Sin embargo, Nussbaum no concibe que el asco pueda tener un lugar dentro de esta concepción.

Es posible que el hecho de que culturalmente exista una tendencia a aceptar el asco desde posturas conservadoras haya ocultado el papel que puede desempeñar en una moralidad de corte liberal. Si esto es así, posiblemente el problema mayor del asco no es su contenido cognoscitivo, puesto que hemos visto que aun cuando a veces no esté justificado, el asco no es necesariamente irracional en todos los casos. Tampoco se trata de su poca confiabilidad, puesto que no necesariamente es ésta 
una emoción menos confiable que otras a las cuales se les asigna algún valor moral. Se trata, en cambio, de sus manifestaciones: existe una tendencia a vincularlo a reclamos relacionados con lo sagrado y con otras nociones que en general no son demasiado compatibles con una sensibilidad moral más liberal, que intenta reemplazar valores sagrados que no necesariamente todos compartan por otros más seculares, más liberales, donde el daño y los derechos adquieren mayor relevancia.

¿Qué dicen las consideraciones precedentes, entonces, sobre el papel que el asco puede tener en la discusión moral de prácticas variadas? En primer lugar, que es una reacción emocional cuya legitimidad moral no debe ser presupuesta; pero además sugieren que no debemos simplificar la cuestión presuponiendo que el asco nunca puede ser moralmente apropiado. Si se quiere avanzar en el debate, debemos desembarazarnos de la concepción sacralizada o conservadora del asco, debemos ir más allá de la idea de que el asco sólo es una reacción emocional legítima dentro de una moralidad conservadora. El tema que tal vez debamos discutir con más cuidado no es si el asco es una emoción de rechazo - porque obviamente lo es-, sino cómo tal rechazo puede desempeñar algún papel en una moralidad basada en los elementos básicos que pensadores como Nussbaum consideran esenciales. ${ }^{13}$

\section{BIBLIOGRAFÍA}

S. Bandes (comp.), 1999, The Pasions of Law, Nueva York University Press, Nueva York/Londres

Bloom, P., 2004, Descartes' Baby, Basic Books, Nueva York.

Brandt, Allan y Paul Rozin (comps.), 1997, Morality and Health, Routledge, Nueva York.

Callahan, D., 1997, "Cloning, the Work Not Done", en Hastings Center Report, vol. 27, no. 5, pp. 18-20.

Cohen, C., 2007, Renewing the Stuff of Life, Oxford University Press, Nueva York.

Darwin, Ch., 1998, The Expression of Emotions in Man and Animals, 3a. ed., Oxford University Press, Nueva York.

De Sousa, R., 1987, The Rationality of Emotion, The MIT Press, Cambridge, Mass.

Glover, J., 2002, Humanity: A Moral History of the Twentieth Century, Yale University Press, Cambridge, Mass.

${ }^{13}$ Agradezco los agudos comentarios de Inmaculada de Melo Martin, Diana Cohen, Maria Julia Bertomeu y Carlos Pereda a versiones anteriores de este trabajo, y las muy útiles sugerencias de los dos árbitros anónimos de la revista. 
Graham, J., J. Haidt y B. Nosek, 2009, "Liberals and Conservatives Rely on Different Sets of Moral Foundations", Journal of Personality and Social Psychology, vol. 96, no. 5, pp. 1029-1046.

Greenspan, P., 1988, Emotions and Reasons, Routledge, Londres.

Haidt, J., 2003, "The Moral Emotions", en R.J. Davidson, K.R. Scherer y H.H. Goldsmith (comps.), Handbook of Afective Sciences, Oxford University Press, Oxford, pp. 852-870.

Haidt, J. y J. Graham, 2007, "When Morality Opposes Justice: Conservatives Have Moral Intuitions that Liberals May Not Recognize", Social Justice Research, vol. 20, no. 1, pp. 98-116.

Haidt, J. y M. Hersh, 2001, "Sexual Morality: The Cultures and Emotions of Conservatives and Liberals", Journal of Applied Psychology, vol. 31, no. 1, pp. 191-221.

Haidt, J., P. Rozin, C. McCauley y S. Imada, 1997, "Body, Psyche, and Culture: The Relationship between Disgust and Morality", Psychology and Developing Societies, vol. 9, no. 1, pp. 107-131.

Hansberg, O., 1996, La diversidad de las emociones, Fondo de Cultura Económica, México.

Hayry, M., 2004, "Another Look at Dignity", Cambridge Quarterly of Healthcare Ethics, vol. 13, pp. 7-14.

Jaggar, A., 1989, "Love and Knowledge: Emotion in Feminist Epistemology", en A. Garry y M. Pearsall (comps.), Women, Knowledge and Reality: Explorations in Feminist Philosophy, Unwin Hyman, Boston, pp. 129-153.

James, W., 1884, "What Is an Emotion?", Mind, vol. 9, no. 34, pp. 188-205.

Kahan, D., 1999, "The Progressive Appropriation of Disgust", en Bandes 1999, pp. 63-79.

Kass, L., 1997, "The Wisdom of Repugnance", The New Republic, vol. 216, no. 22, pp. 17-26.

Kekes, J., 1992, "Disgust and Moral Taboos", Philosophy, vol. 67, no. 262, pp. 431-446.

Little, M.O., 1995, "Seeing and Caring: The Role of Affect in Feminist Moral Epistemology", Hypatia, vol. 10, no. 3, pp. 117-137.

Meyers, D., 1993, "Moral Reflection: Beyond Impartial Reason", Hypatia, vol. 8 , no. 3, pp. 21-47.

_ 1997 , "Emotion and Heterodox Moral Perception: An Essay in Moral Social Psychology", en D. Meyers (comp.), Feminists Rethink the Self, Westview, Nueva York, pp. 197-218.

Midgley, M., 2000, "Biotechnology and Monstrosity", Hastings Center Report, vol. 30 , no. 5 , pp. 7-15.

Miller, W.I., 1998, "Sheep, Joking, Cloning and the Uncanny", en M. Nussbaum y C. Sunstein (comps.), Clones and Clones: Facts and Fantasies about Human Cloning, W.W. Norton, Nueva York, pp. 78-87.

— 1997, The Anatomy of Disgust, Harvard University Press, Cambridge.

Nelson Lindemann, J., 2003, Hippocrates' Maze: Ethical Explorations of the Medical Labyrinth, Rowman and Littlefield, Lanham. 
Nussbaum, M., 2004, Hiding from Humanity, Princeton University Press, Princeton.

— 2001, Upheavals of Thought, Cambridge University Press, Cambridge.

_ 1999 , "Secret Sewers of Vice: Disgust, Bodies and the Law", en Bandes 1999, pp. 19-62.

Prinz, J., 2007, The Emotional Construal of Morals, Oxford University Press, Nueva York.

_ - 2004, Gut Reactions: A Perceptual Theory of Emotion, Oxford University Press, Nueva York.

Rozin, P., 1997, "Moralization", en Brandt y Rozin 1997, pp. 379-401.

Rozin, P., J. Haidt, y C.R. McCauley, 1999, "Disgust: The Body and Soul Emotion", en T. Dalgleish y M. Power (comps.), Handbook of Cognition and Emotion, John Wiley, Sussex, pp. 429-445.

_, 1993 , "Disgust", en M. Lewis y J. Haviland (comps.), Handbook of Emotions, Guilford, Nueva York, pp. 575-594.

Rozin, P., L. Lowery, S. Imada y J. Haidt, 1999, "The CAD Triad Hypothesis: A Mapping between Three Moral Emotions (Contempt, Anger, Disgust) and Three Moral Codes (Community, Autonomy, Divinity)", Journal of Personality and Social Psychology, vol. 76, no. 4, pp. 574-586.

Rozin, P. y A.E. Fallon, 1987, "A Perspective on Disgust", Psychological Review, vol. 94, no. 1, pp. 23-41.

Sherman, N., 1989, The Fabric of Character, Oxford University Press, Nueva York.

Shweder, R., N. Much, M. Mahapatra y L. Park, 1997, “The 'Big Three' of Morality (Autonomy, Community and Divinity) and the 'Big Three' Explanations of Suffering", en Brandt y Rozin 1997, pp. 119-169.

Solomon, R., 1993, The Passions: Emotions and the Meaning of Life, Hackett, Indianápolis.

_ 1976 , The Passions, Doubleday, Nueva York.

Recibido el 10 de febrero de 2009; aceptado el 14 de octubre de 2009. 